\title{
Canagliflozin Facilitates Reverse Cholesterol Transport Through Activation of AMPK/ABC Transporter Pathway
}

\author{
Yingnan Zhao ${ }^{1,2}$ \\ Yanping $\mathrm{Li}^{2}$ \\ Qinhui Liu' \\ Qin Tang ${ }^{1,2}$ \\ Zijing Zhang ${ }^{1,2}$ \\ Jinhang Zhang ${ }^{1,2}$ \\ Cuiyuan Huang ${ }^{1,2}$ \\ Hui Huang ${ }^{1,2}$ \\ Guorong Zhang ${ }^{1,2}$ \\ Jian Zhou ${ }^{1,2}$ \\ Jiamin Yan ${ }^{1,2}$ \\ Yan Xia ${ }^{1,2}$ \\ Zhiyong Zhang ${ }^{1,2}$ \\ Jinhan $\mathrm{He}^{1,2}$ \\ 'Department of Pharmacy, National \\ Clinical Research Center for Geriatrics, \\ West China Hospital, Sichuan University, \\ Chengdu, Sichuan Province, People's \\ Republic of China; ${ }^{2}$ Laboratory of Clinical \\ Pharmacy and Adverse Drug Reaction, \\ National Clinical Research Center for \\ Geriatrics, West China Hospital, Sichuan \\ University, Chengdu, Sichuan Province, \\ People's Republic of China
}

Correspondence: Zhiyong Zhang; Jinhan He Department of Pharmacy, National Clinical Research Center for Geriatrics, West China Hospital, Sichuan University, Chengdu, Sichuan Province, People's Republic of China $\mathrm{Tel} / \mathrm{Fax}+86-28-85426416$

Email asg-yong@I63.com; jinhanhe@scu. edu.cn
Background and Purpose: Cholesterol is an essential lipid and its homeostasis is a major factor for many diseases, such as hyperlipidemia, atherosclerosis, diabetes, and obesity. Sodium-glucose cotransporter 2 (SGLT2) inhibitor canagliflozin (Cana) is a new kind of hypoglycemic agent, which decreases urinary glucose reabsorption and reduces hyperglycemia. Cana has been shown to regulate serum lipid, decrease serum triglyceride and increase serum high-density lipoprotein-cholesterol (HDL-C), and improve cardiovascular outcomes. But evidence of how Cana impacted the cholesterol metabolism remains elusive.

Methods: We treated Cana on mice with chow diet or western diet and then detected cholesterol metabolism in the liver and intestine. To explore the mechanism, we also treated hepG2 cells and Caco2 cells with different concentrations of Cana.

Results: In this study, we showed that Cana facilitated hepatic and intestinal cholesterol efflux. Mechanically, Cana via activating adenosine monophosphate-activated protein kinase (AMPK) increased the expression of ATP-binding cassette (ABC) transporters ABCG5 and ABCG8 in liver and intestine, increased biliary and fecal cholesterol excretion.

Conclusion: This research confirms that Cana regulates cholesterol efflux and improves blood and hepatic lipid; this may be a partial reason for improving cardiovascular disease.

Keywords: canagliflozin, ATP-binding cassette (ABC) transporters G5/8, cholesterol efflux, AMPK

\section{Introduction}

Cholesterol, as an important biologically active substance, plays a significant role in maintaining membrane structure, biosynthesis of bile acid and steroid hormones. ${ }^{1}$ Cholesterol homeostasis dysregulated is also involved in the pathophysiology of various diseases, such as hyperlipidemia, cardiovascular disease, ${ }^{2}$ obesity $^{3}$ and diabetes. $^{4}$

Cholesterol homeostasis mainly involves absorption of cholesterol in the intestine or biosynthesis in the liver, reverse cholesterol transport, converse into bile acid, and biliary and fecal excretion. Intestinal absorption is mainly mediated by Niemann-Pick C1-Like 1(Npc111). ${ }^{5}$ 3-hydroxy-3-methylglutarylCoA reductase (Hmgcr) is a rate-limiting enzyme for sterol biosynthesis. Cholesterol mainly reverses transport into the liver by high-density lipoprotein (HDL); then, a third of them are converted into bile acid through the classical pathway (mainly by Cyp7a1 and Cyp8b1) or alternative pathways (mainly by Cyp27a1 and Cyp7b1). ${ }^{6}$ Cholesterol transport is mediated by many membrane 
transporters, including ATP-binding cassette (ABC) transporters Abcal and Abcg1. Abcal promotes cholesterol export to Apo A-I, and Abcg1 plays a role in cholesterol export to HDL. ${ }^{1}$ In the liver and intestine, Abcg5/8 promotes secretion of cholesterol into bile or gut lumen. It has been reported that increased cholesterol excretion but not absorption can improve cardiovascular diseases. ${ }^{7}$ The liver $\mathrm{X}$ receptor (LXR) is an important regulator of cholesterol homeostasis. ${ }^{8}$ LXR regulated expression of the $\mathrm{ABC}$ transporters Abca1, Abag1 and Abcg5/8 is critical in enhancing cholesterol efflux. $^{9}$

AMPK is an important cellular energy sensor; a number of recent studies have shown that AMPK has a wide range of biological roles, involving mitochondrial oxidative respiration, energy homeostasis and autophagy. ${ }^{10}$ And it has the potential to affect many human diseases, such as cancer, type 2 diabetes and atherosclerosis. ${ }^{10,11}$ A number of drugs, like salicylate ${ }^{12}$ and Pueraria, ${ }^{13}$ activating AMPK, have been reported to stimulate cholesterol efflux from macrophage and decrease cellular lipid accumulation via AMPK-Abcal axis. Activation of AMPK increased the expression of Abcal and its transcriptional activator LXR $\alpha$ in macrophages. ${ }^{14}$ And metformin, a classical AMPK activating drug, increases Abcg5 and Abcg8 expression in the liver via regulating the stability of Period 2 which is a repressor of transcription on the Abcg8 promoter. ${ }^{15}$ Thus, AMPK plays an important role in cholesterol homeostasis.

Canagliflozin (Cana), one of the sodium glucose cotransporters (SGLT) inhibitors, inhibits the reabsorption of glucose in the proximal glomerular tubules. Except reducing hyperglycemia, clinical trials and experience in mice have confirmed that Cana reduces body weight, ${ }^{16,17}$ lowers blood lipid, ${ }^{18}$ and reduces the risk of cardiovascular events. ${ }^{19-21}$ However, there is still a lack of evidence as to how Cana impacted the cholesterol metabolism.

In this study, we showed that Cana can facilitate the cholesterol efflux into bile and feces through upregulating Abcg5 and Abcg8 expression in the liver and ileum. In vitro, we found that Cana could directly activate AMPK pathway and upregulate the expression of Abcg5 and Abcg8. Inhibition of AMPK pathway abolished the effect of Cana on the regulation of Abcg5/8. Our study provides a plausible explanation of Cana's effect on cholesterol metabolism.

\section{Materials and Methods}

\section{Animal Model and Treatment}

C57BL/6J male mice ( 8 weeks old) were divided into four groups $(\mathrm{n}=8)$ and, respectively, fed with chow diet or western diet $(42 \%$ of kcal from fat, $0.5 \%$ cholesterol, Medicinence, Cat, \#TD88137A) for 12 weeks. Canagliflozin (MB1516, Meilunbio ${ }^{\circledR}$, Dalian, China) (Supplementary Figure 1) was mixed with chow diet or western diet at $0.03 \%(\mathrm{w} / \mathrm{w}){ }^{22}$ Fecal samples and urine were collected for three consecutive days using metabolic cages before being killed. Mice were killed through $\mathrm{CO}_{2}$ after fasted overnight. Blood, liver, duodenum, jejunum and ileum were collected and stored in $-80^{\circ} \mathrm{C}$. All mice were housed in the institutional animal care of West China Hospital of Sichuan University with $24^{\circ} \mathrm{C}$-indoor temperature, $55 \pm 15 \%$ relative humidity, and a 12 -hour light/dark cycle. Mice were bred in standard cage with ad libitum feeding. All animal experiments in this research were carried out in accordance with the relevant regulations of the Sichuan University Laboratory Animal Ethics committee and approved by the Institutional Review Board (or Ethics Committee) of the Sichuan University Laboratory Animal Ethics committee (2020061A).

\section{Histological Staining}

Mouse livers were fixed and embedded in paraffin, sectioned at $4 \mu \mathrm{m}$, and stained with hematoxylin and eosin (H\&E). Oil-Red-O staining was performed by $10 \mu \mathrm{m}$ frozen sections. The samples were examined under a light microscope (Nikon, Tokyo, Japan), at 200X or 400X magnification.

\section{Serum Alanine Transaminase (ALT), Aspartate Aminotransferase (AST) Assays} Blood was held at $4^{\circ} \mathrm{C}$ for 2 hours, then centrifuged at $6000 \mathrm{rpm}$ for 10 minutes, and supernatant removed. The serum was stored at $-80^{\circ} \mathrm{C}$. Serum ALT $(100,020,000$, Biosino Bio) and AST (100,020,010, Biosino Bio) levels were measured using appropriate enzymatic kits.

\section{Cholesterol, Triglycerides, Glucose and Bile Acid Assays}

Mice feces of 24 hours were collected for three consecutive days. Then dried and ground the feces, extracted with $75 \%$ ethanol at 55 degrees Celsius for 2 hours, centrifuged at $1500 \mathrm{rpm}, 10 \mathrm{~min}$ and took the supernatant for 
detection. Liver lipids were extracted with chloroform and dissolved with $1 \%$ triton ethanol. Serum, hepatic and fecal lipids were detected by the enzymatic kits (Biosino, Beijing, China). Urine was diluted 100 times with PBS, then detected glucose with an assay kit $(100,020,100$, Biosino, Beijing, China). Blood glucose was measured using a glucometer (Roche, Basel, Switzerland).

\section{Cell Culture and Treatments}

All cell lines were cultured under standard conditions at $37^{\circ} \mathrm{C}, 5 \% \mathrm{CO}_{2}$ in a humidified incubator. HepG2 cells (ATCC, HB8065) and Caco2 cells (ATCC, CRL-2102) were maintained in a medium (DMEM, supplemented with $10 \%$ fetal bovine serum). Cells were cultured in a 12 well, and were pretreated with $10 \mu \mathrm{M}$ Compound C (Merck, 866,405-64-3) for $1 \mathrm{~h}$, then were treated with $10 \mu \mathrm{M}$ or $30 \mu \mathrm{M}$ Cana for $2 \mathrm{~h}$ to detect the expression of AMPK expression, or $24 \mathrm{~h}$ to detect the expression of ABCG5/8.

\section{Western Blotting Analysis}

The total protein was extracted by loading buffer and used BCA method to ensure protein concentration. Primary antibodies and fluorescence secondary antibodies were listed in Supplementary Table 1. The signal was visualized using LI-COR System (Lincoln, NE). Quantitative determination of band intensity was analyzed using Image Studio (Li-COR).

\section{Total RNA Extraction and Real-Time PCR Analysis}

The total RNA of the tissues was extracted with trizol reagent (135,306, Life Technologies). The cDNA synthesized from RNA by reverse transcription kit (RR037A; TaKaRa, Kyoto, Japan) and cDNA samples were quantified by CFX96 Real-time RT-PCR System (Hercules, CA) with SYBR Green PCR Master Mix (Takara, Tokyo, Japan). The Primers are listed in Supplementary Table 2.

\section{Statistical Analysis}

Data were shown as mean \pm SEM. Student's $t$-test or oneway ANOVA Tukey's test was used for data analysis. In animal experiments, there are 8 mice in each group; and in the cell experiments, there are three duplicate holes in each group. $\mathrm{P}<0.05$ was considered statistically significant.

\section{Results}

Cana Improved Serum Lipid Profiles and Hepatic Lipid Metabolism

It has been reported that Cana can improve lipid metabolism. ${ }^{19}$ In western diet-fed mice, Cana treatment significantly decreased western diet increased the body weight $\quad(35.41 \pm 1.244$ to $30.47 \pm 0.5775 \mathrm{~g}, \quad \mathrm{p}=0.0026)$ (Supplementary Figure 2A and Supplementary Table 3). Compared with control, Cana slightly increased the food intake, but did not reach statistical difference (Supplementary Figure 2B, Supplementary Table 3). As expected, Cana decreased the blood glucose under both chow diet and western diet $(186.1 \pm 5.516$ to $136.6 \pm 4$.$569 \mathrm{mg} / \mathrm{dl}, \mathrm{p}<0.0001$ ) (Supplementary Figure 2C, Supplementary Table 3). The glucose level in urine was significantly increased by $6000-8000$ times in Cana treated mice (Supplementary Figure 2D, Supplementary Table 3).

We then evaluated the effect of Cana on the lipid metabolism in mice fed with chow and western diet. Serum cholesterol level was significantly decreased from $280.5 \pm 15.92 \mathrm{mg} / \mathrm{dl}$ to $217.1 \pm 20.98 \mathrm{mg} / \mathrm{dl}(\mathrm{p}=0.0275)$ in Cana treated mice (Figure 1A). Further analysis revealed that the lower serum LDL-C could account for the decreased serum cholesterol seen in Cana treated mice (Figure 1B). Unexpectedly, Cana increased the HDL-C under both chow diet and western diet (Figure 1C). The serum level of triglyceride was also lowered from in Cana treated mice after western diet (Figure 1D). We went on to evaluate the effect of Cana on hepatic lipid metabolism. $\mathrm{H} \& \mathrm{E}$ staining and Oil red $\mathrm{O}$ staining indicated that hepatic lipid was less in Cana treated mice (Figure 1E and F). Lipid quantification revealed that the levels of cholesterol and triglyceride were significantly lower in Cana treated mice under western diet (Figure $1 \mathrm{G}$ and $\mathrm{H}$ ).

\section{Cana Upregulated Hepatic Cholesterol Transport}

To understand the cause of Cana regulated lipid metabolism, we measured the mRNA expression of genes involved in cholesterol metabolism. We first detected the gene expression in cholesterol biosynthesis. Cana had little effect on the expression of Hmgcr, and slightly decreased the expression Hmgcs in the liver of mice fed with chow diet (Supplementary Figure 3A). However, the expressions of these two genes were significantly decreased in Cana treated mice (Figure 2A). Once 


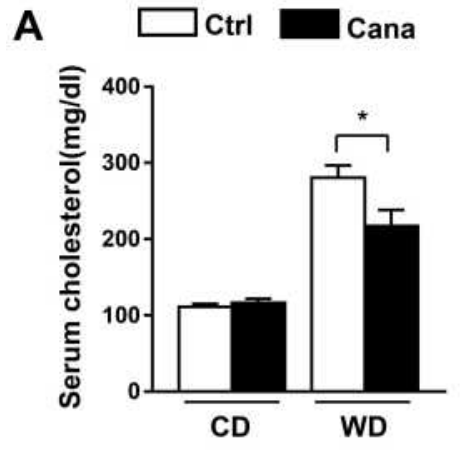

E
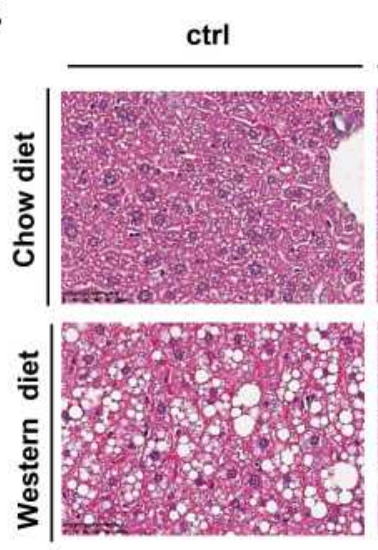

B

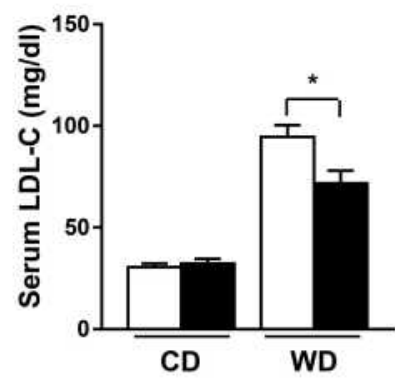

C

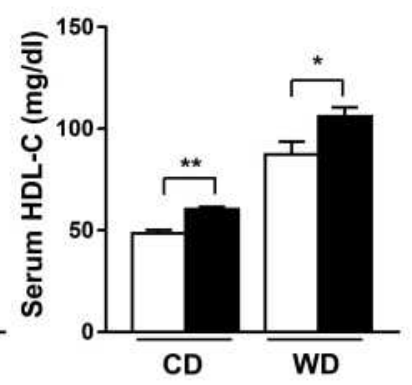

D

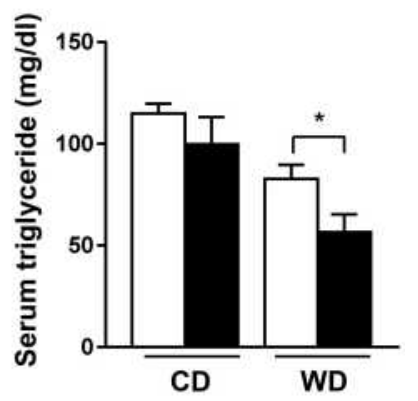

$\mathbf{F}$
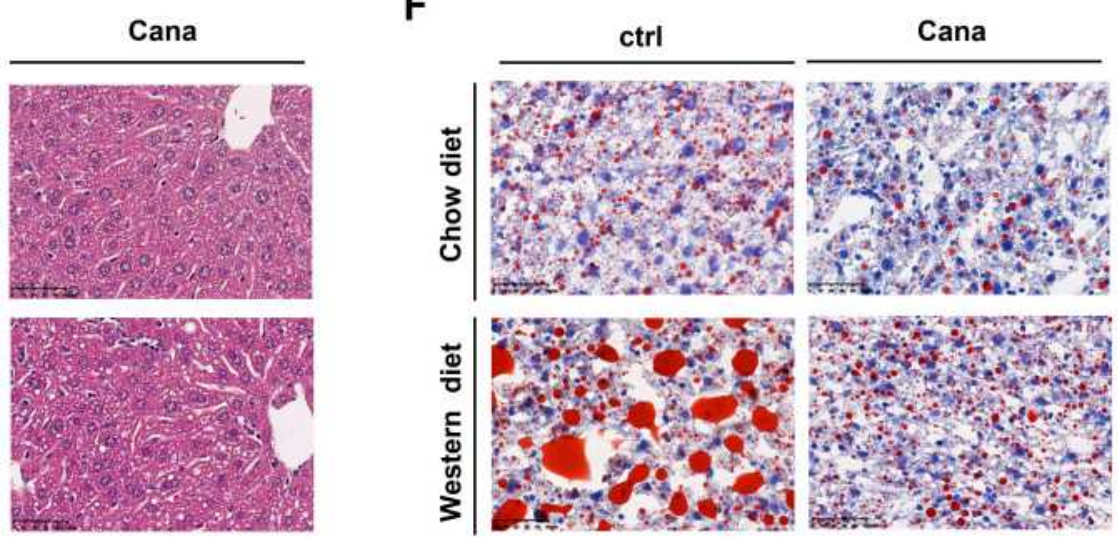

G

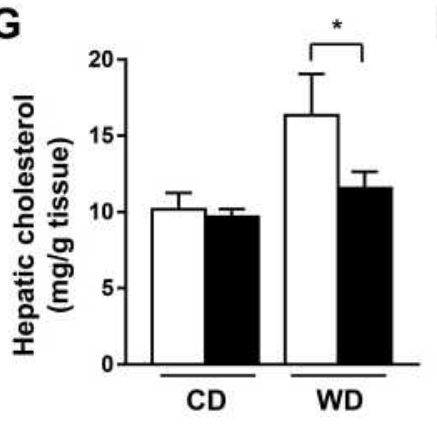

H

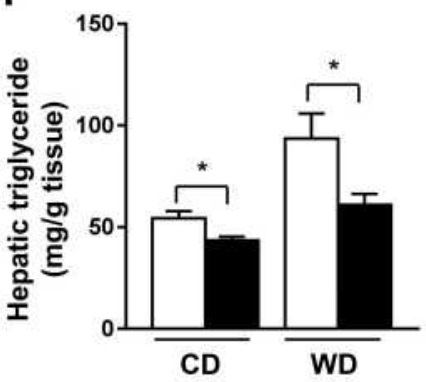

Figure I Serum parameters and hepatic lipid profile in Cana treated mice. 8 weeks old C57BL/6) mice were fed with chow diet or western diet ( $\mathrm{n}=8)$ for I 2 weeks $(0.03 \%$ w/w Cana was mixed with diet). Serum levels of (A) total cholesterol, (B) LDL-C, (C) HDL-C and (D) TG in mice were shown. (E) H\&E staining (X 400) and (F) Oil Red O staining $(X 400)$ of liver tissues were shown. Hepatic $(\mathbf{G})$ cholesterol and $(\mathbf{H})$ triglyceride levels were also represented. Data was shown with mean \pm SEM. $*$ P $<0.05$, $* * \mathrm{P}<0.01$ compared with control.

Abbreviations: HDL-C, HDL-cholesterol; LDL-C, LDL-cholesterol; TG, triglyceride.

synthesized, cholesterol can be converted into bile acid, or transported into bile by its transporters. ${ }^{6}$ The conversion of cholesterol into bile acid is controlled through the classical pathway (main enzyme are Cyp7a1 and Cyp8b1) and alternative pathway (main enzyme are Cyp27a1 and Cyp7b1). ${ }^{6}$ The mRNA expressions of these bile acid synthetic genes were significantly increased by 1.5-2.5 times in Cana treated mice upon western diet (Figure 2B). On chow diet, the expressions of Cyp7a1 and Cyp27a1 were also increased
(Supplementary Figure 3B). Cholesterol can be transported into plasma by Abcal and Abcg1, or into bile by Abcg5 and Abcg8. ${ }^{23}$ The mRNA expression of these transporters was upregulated about 1.5 times by Cana on both chow diet and western diet (Figure 2C and Supplementary Figure 3C). Consistently, the protein levels of Abcg5 and Abcg8 were increased by 1.5-2 times in Cana treated mice (Figure 2D and E). Consequently, the biliary concentration of cholesterol and bile acid was higher in Cana treated mice 
A

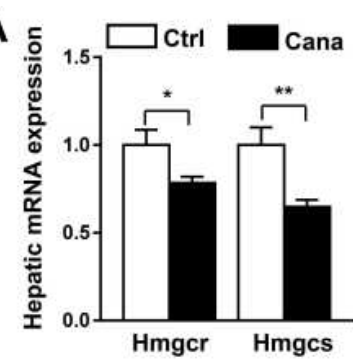

C

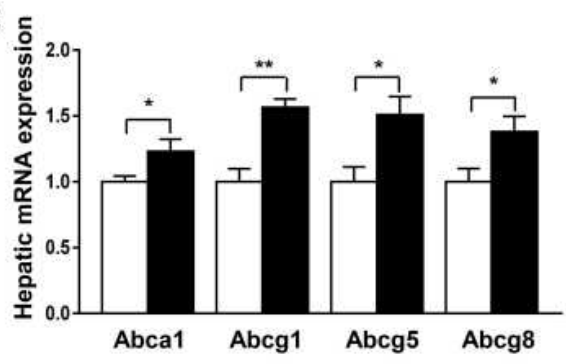

F

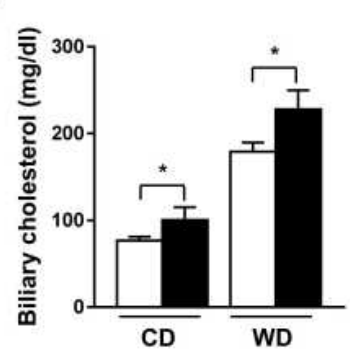

H

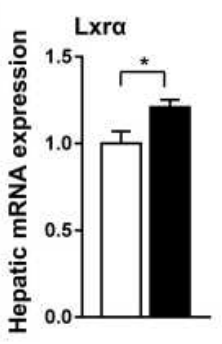

G

I
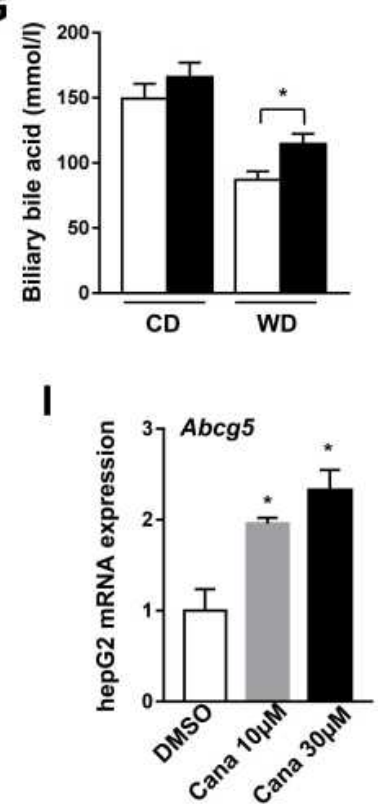

Cana

K

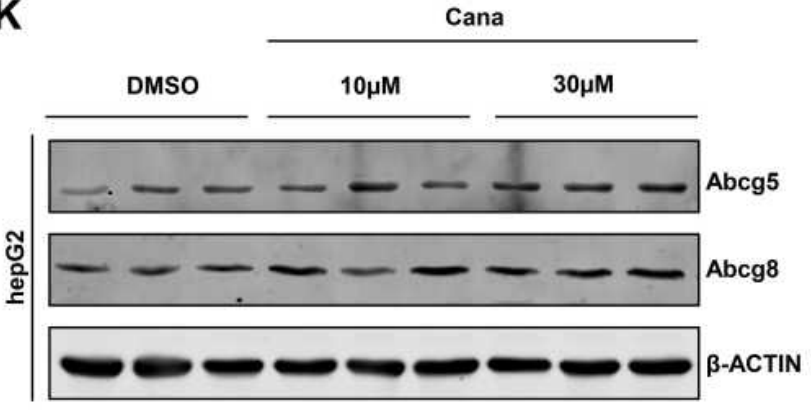

J
B

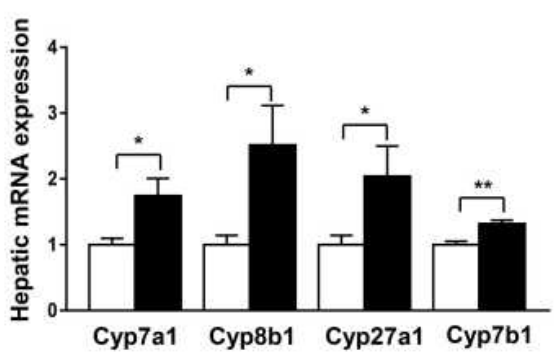

D

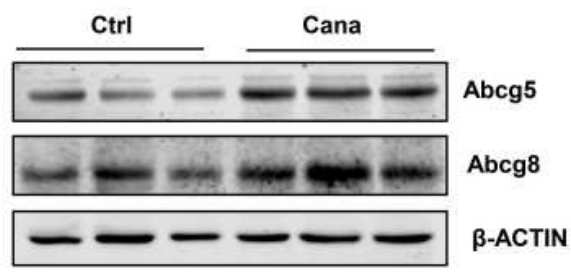

E
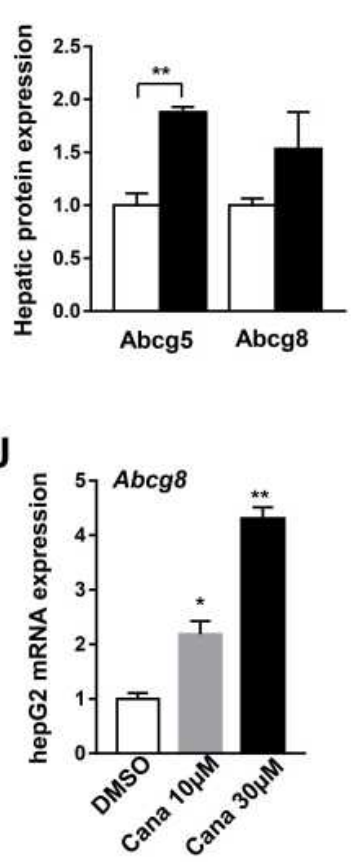

L

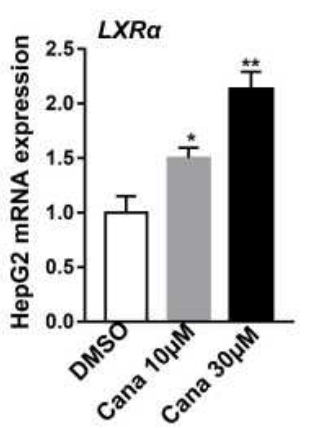

Figure 2 Cana upregulated hepatic cholesterol transport. 8 weeks old C57BL/6] mice were fed with western diet for I2 weeks. (A) Real-time PCR analysis of hepatic mRNA levels of cholesterol biosynthesis, (B) bile acid synthesis from cholesterol and (C) cholesterol efflux $(n=6-8)$ in western diet fed mice. (D) Western blot analysis hepatic protein expression of ABCG5 and ABCG8. (E) Quantification of ABCG5/8 protein were shown. Biliary concentrations of (F) cholesterol and (G) bile acid in chow diet and Western diet fed mice were measured $(n=5-7)$. (H) The mRNA levels of $L X R \alpha$ was detected $(n=8)$. HepG2 cells were treated with Cana I0 $\mu M$ or $30 \mu M$ for $24 h$, the mRNA expression of (I) ABCG5 and (J) ABCG8 were detected by real-time PCR $(n=3)$, (K) the protein expression of $A B C G 5 / 8$ were measured by Western Blot. (L) The mRNA expression of $L X R \alpha$ was analyzed in hepG2 cells $(n=3)$. The data was shown with mean $\pm S E M$. *P $<0.05$, **P<0.0I compared with control or DMSO group. 
(Figure 2F and $\mathrm{G}$ ). In the liver, LXR is involved in transcriptional control of Cyp7A1, as well as induction of Abcal and Abcg5/8 expression, and thus accelerates biliary cholesterol disposal. ${ }^{24}$ The mRNA expression of LXR in liver is increased by Cana on chow diet (Supplementary Figure 3D) and western diet (Figure 2H). To test whether Cana could directly regulate the expression of Abcg5 and Abcg8, we treated HepG2 cells with different concentrations of Cana. We found that Cana could increase the mRNA expression of Abcg5 and Abcg8 more than 2 times (Figure 2I and J). The protein levels of Abcg5 and Abcg8 were also upregulated about 1.5 times by Cana (Figure $2 \mathrm{~K}$ and Supplementary Figure $\underline{3 \mathrm{E} \text { and } \mathrm{F}}$ ). The mRNA expression of LXR in hepG2 cells also increased about 1.5 times by treatment with low dose of Cana and more than 2 times by high dose of Cana (Figure 2L).

\section{Cana Augment the Intestinal Abcg5/8 Expression}

Intestinal cholesterol absorption and transport also play a vital role in cholesterol homeostasis. ${ }^{25}$ On the apical, cholesterol is transported into the enterocytes by Npc111. ${ }^{5}$ Cana treatment had little effect on the expression of Npc111 (Figure 3A). Cholesterol in the enterocytes either transports back into the intestinal lumen by abcg $5 / 8$ or transports into basolateral by Abcal and Abcg1. ${ }^{26}$ The expression of Abcg5/8 was upregulated 2-3 times than that in the Cana treated duodenum and jejunum (Figure 3A), suggesting higher efflux of cholesterol back to the lumen. The expressions of Abcal and Abcg1 were also higher 1.5-2 times in the duodenum and jejunum (Figure 3B). Apo A-I (apolipoprotein A-I) receives cholesterol efflux from Abca1, then participates in new HDLs synthesis. ${ }^{27}$ The expression of Apo A-I was consistently increased in the duodenum and jejunum of Cana treatment western diet fed mice (Figure 3B). As a result of elevated efflux of cholesterol, the fecal cholesterol was consistently higher in Cana treated mice (Figure 3C). LXR agonist upregulates Abca1, Abag1 and Abcg5/8 expression, and accelerates biliary and fecal cholesterol disposal. ${ }^{24,28}$ The mRNA expression of LXR was also upregulated in intestine (Figure 3D). We also tested the effect of Cana in human colon adenocarcinoma cell line, Caco 2 cells. In Caco 2 cells, both mRNA and protein expression of Abcg5 and Abcg8 were higher about 1.5-2 times after Cana treatment (Figure 3F-H, Supplementary Figure 4A and B). And the LXR expression was also significantly increased by about 2.5 times by Cana (Figure 3E).

\section{Cana Increased Abcg5 and Abcg8 Expression via Active AMPK}

To understand the mechanism of Cana regulated Abcg5 and Abcg8 expression, we explored the signaling pathway. Cana has been reported to activate AMPK in several cell lines. ${ }^{29,30}$ Western blot result showed that Cana could active AMPK in liver tissue (Figure 4A) and duodenum and jejunum (Supplementary Figure 5). In hepG2 cells, Cana could directly activate AMPK and its downstream phosphorylation of ACC (Figure 4B). In $\mathrm{Caco} 2$ cells, AMPK was also activated by Cana treatment (Figure 4C). To confirm whether Cana regulated Abcg5 and Abcg8 was AMPK dependent, we treated hepG2 and Caco2 cells with compound $\mathrm{C}$, a pharmacological inhibitor of AMPK. Inhibition of AMPK pathway abolished Cana increased expression of Abcg5 and Abcg8 in both hepG2 and Caco2 cells (Figure 5A-F, Supplementary Figure 6A-F). Cana upregulated expressions of LXR in both hepG2 and $\mathrm{Caco} 2$ cells were also inhibited by Compound C (Supplementary Figure 6G and H). Taken together, Cana increased Abcg5 and Abcg8 expression via activating AMPK pathway.

\section{Discussion and Conclusion}

Cana, the first SGLT2 inhibitor, was approved as a hypoglycemic drug by the FDA in $2013 .{ }^{18}$ It increases urinary sugars excretion by inhibiting glucose reabsorption in proximal glomerular tubules. Cana has been reported to regulate cholesterol metabolism. Our study reported that Cana upregulated HDL-C, and downregulated the level of cholesterol and triglyceride in serum. This result that Cana downregulated cholesterol and triglyceride was also shown in APOE-/- mice with high cholesterol feeding. ${ }^{31}$ Yu et al study showed that Cana decreased cholesterol in serum but had little effect on triglyceride in C57Bl/6NTac mice fed with a high-fat diet and injected with streptozotocin. ${ }^{32}$ Basu et al also analyzed the lipid profile in serum of diabetic CETP-Apolipoprotein B100 transgenic mice, and their results showed that Cana increased LDL-C and HDL$\mathrm{C}$ and reduced TG in serum. ${ }^{33}$ The different effect of Cana is likely a result of different backgrounds of mice and diet feeding.

Cholesterol homeostasis in the body is mainly involved in de novo synthesis, intestinal absorption, and biliary and 


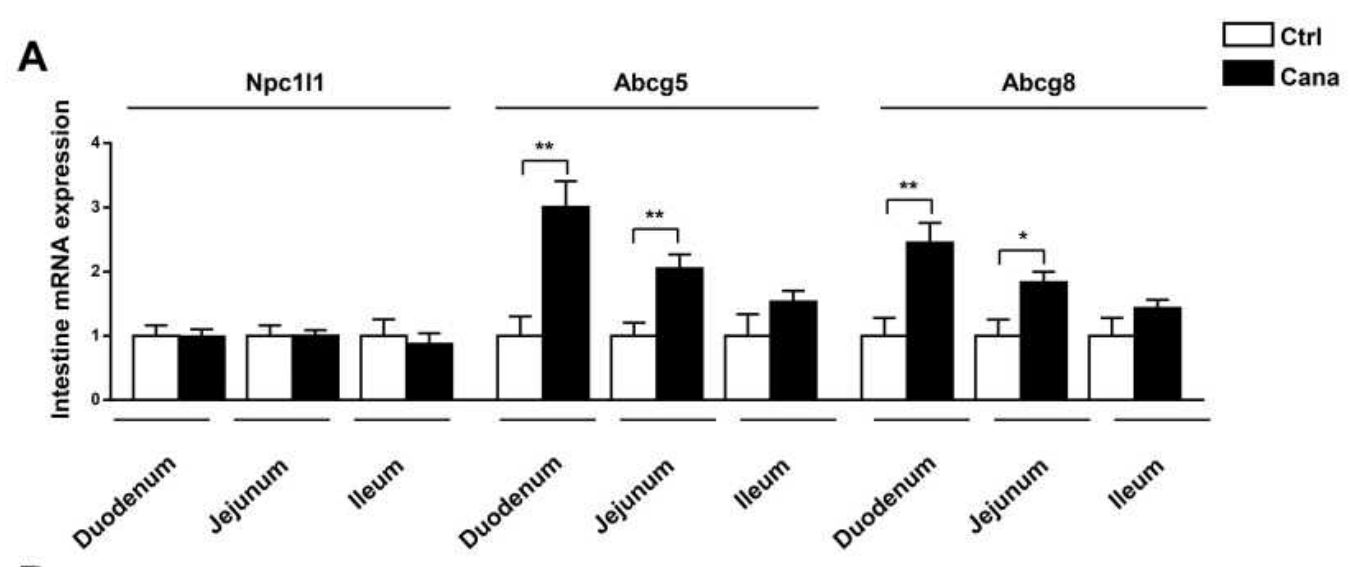

B

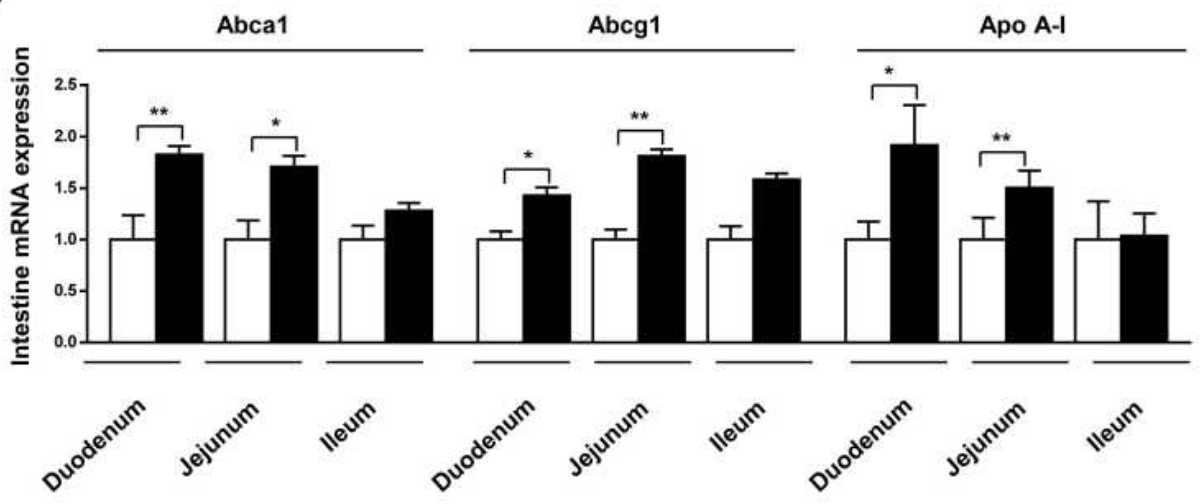

C

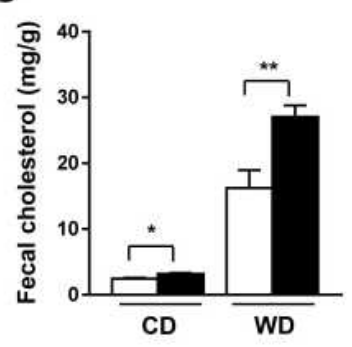

D

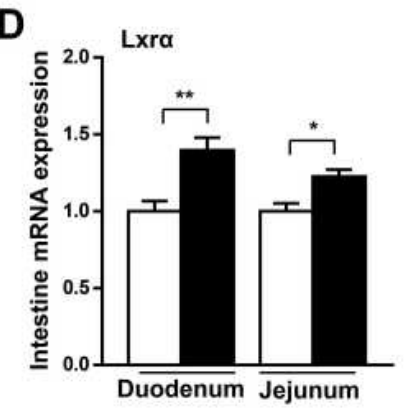

E

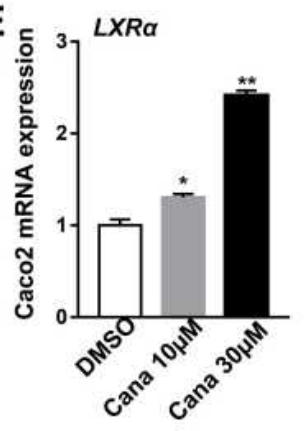

$\mathbf{F}$

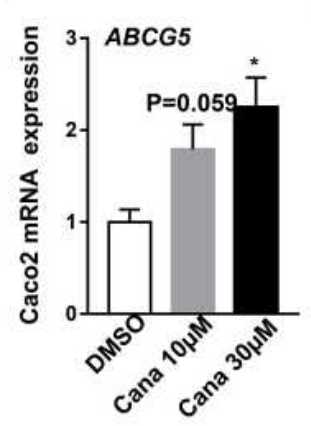

G

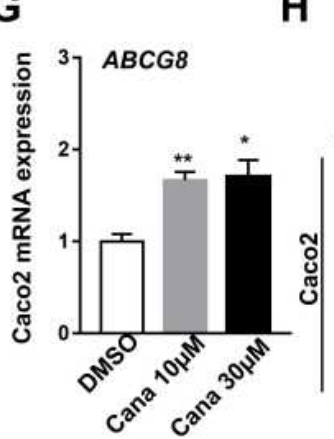

H

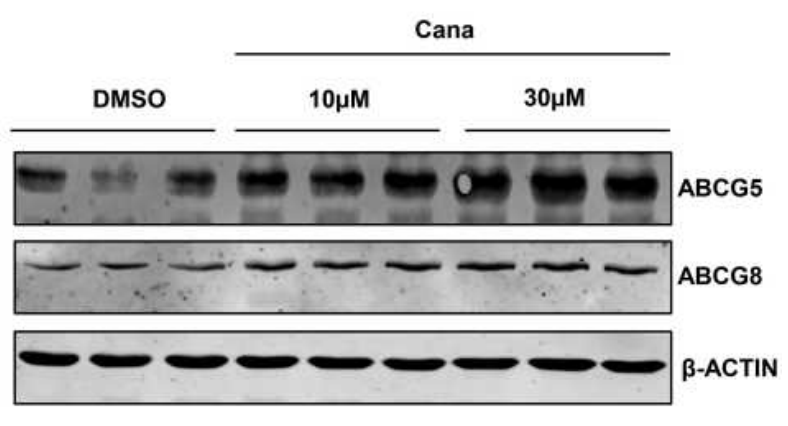

Figure 3 Cana increases expression of intestinal Abcg5 and Abcg8. 8 weeks old C57BL/6] mice were fed with western diet for I2 weeks. (A) Real-time PCR analysis of cholesterol absorption and efflux in duodenum, jejunum and ileum, (B) and mRNA expression of HDL-cholesterol transports ( $\mathrm{n}=5-8)$. (C) Fecal cholesterol of chow diet and Western diet fed mice were measured $(n=6-8)$. (D) The mRNA expression of $L X R \alpha$ in duodenum and jejunum was detected with real-time PCR. Caco2 cells were treated with canagliflozin $10 \mu \mathrm{M}$ or $30 \mu \mathrm{M}$ for $24 \mathrm{~h}$. The mRNA levels of $(\mathbf{E}) L X R \alpha,(\mathbf{F}) A B C G 5$ and $(\mathbf{G}) A B C G 8$ were measured $(\mathrm{n}=3)$. (H) Western blot analyzed the $A B C G 5$ and $A B C G 8$ protein level of $\mathrm{Caco}$. The data was shown with mean $\pm \mathrm{SEM}$. $* \mathrm{P}<0.05,{ }^{*} * \mathrm{P}<0.01$ compared with control or DMSO group. 
A

A

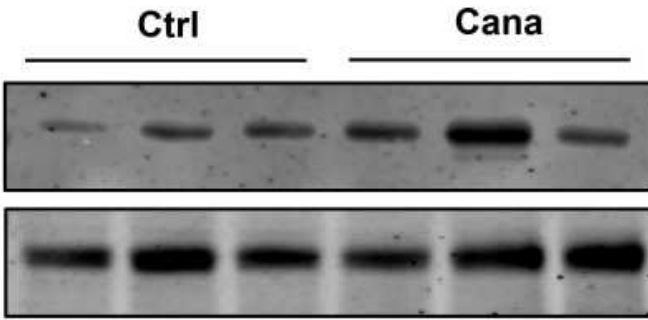

$\longrightarrow$

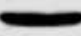

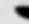

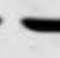

p-AMPK

AMPK

$\beta-A C T I N$
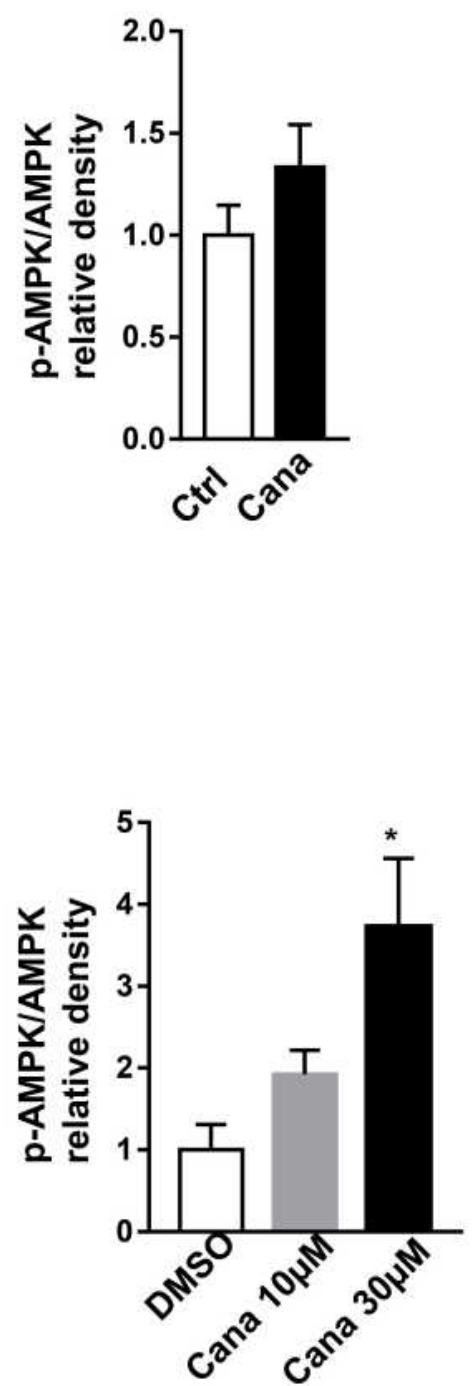

C

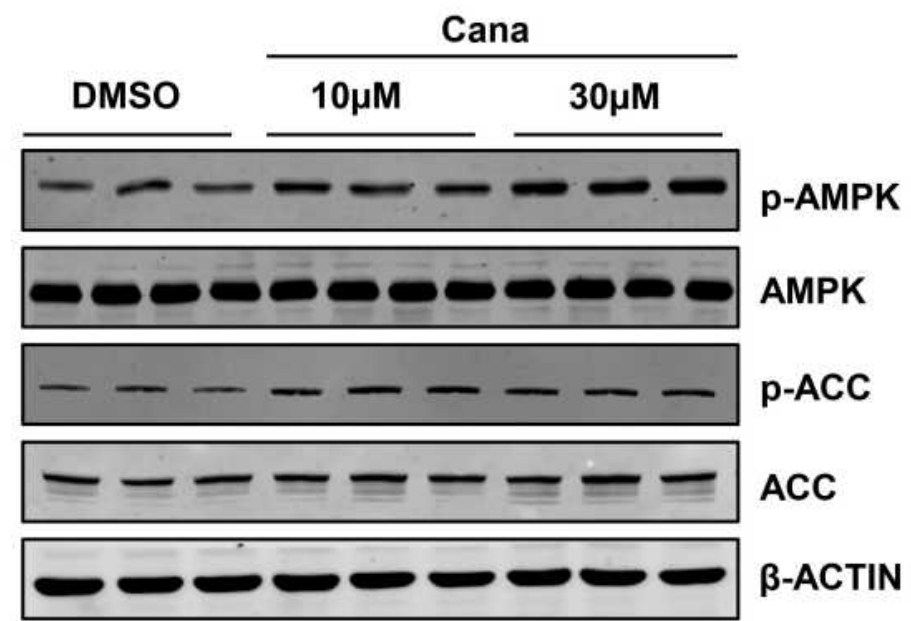

p-AMPK

AMPK

p-ACC

ACC

$\beta$-ACTIN

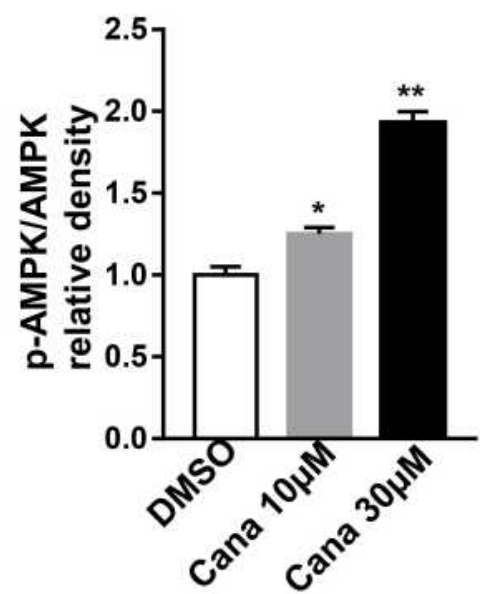

Figure 4 Cana activated AMPK in vivo and vitro. (A) Hepatic protein expression of p-AMPK and AMPK in Western diet fed mice were measured and the p-AMPK/AMPK ratio was shown. HepG2 cells and Caco2 cells were treated with Cana $(10 \mu M$ or $30 \mu \mathrm{M})$ for $2 \mathrm{~h}(\mathrm{n}=3)$. P-AMPK and p-ACC of $(\mathbf{B})$ hepG2 and $(\mathbf{C})$ Caco2 cells were analyzed. And the quantification of $\mathrm{p}$-AMPK/AMPK were revealed. The data was shown with mean $\pm \mathrm{SEM}$. *P<0.05, $* * \mathrm{P}<0.01$ compared with control or DMSO group.

Abbreviations: AMPK, adenosine monophosphate activated protein kinase; p-AMPK, phosphorylation-AMPK; p-ACC, phosphorylation-acetyl-coenzyme A carboxylase. 

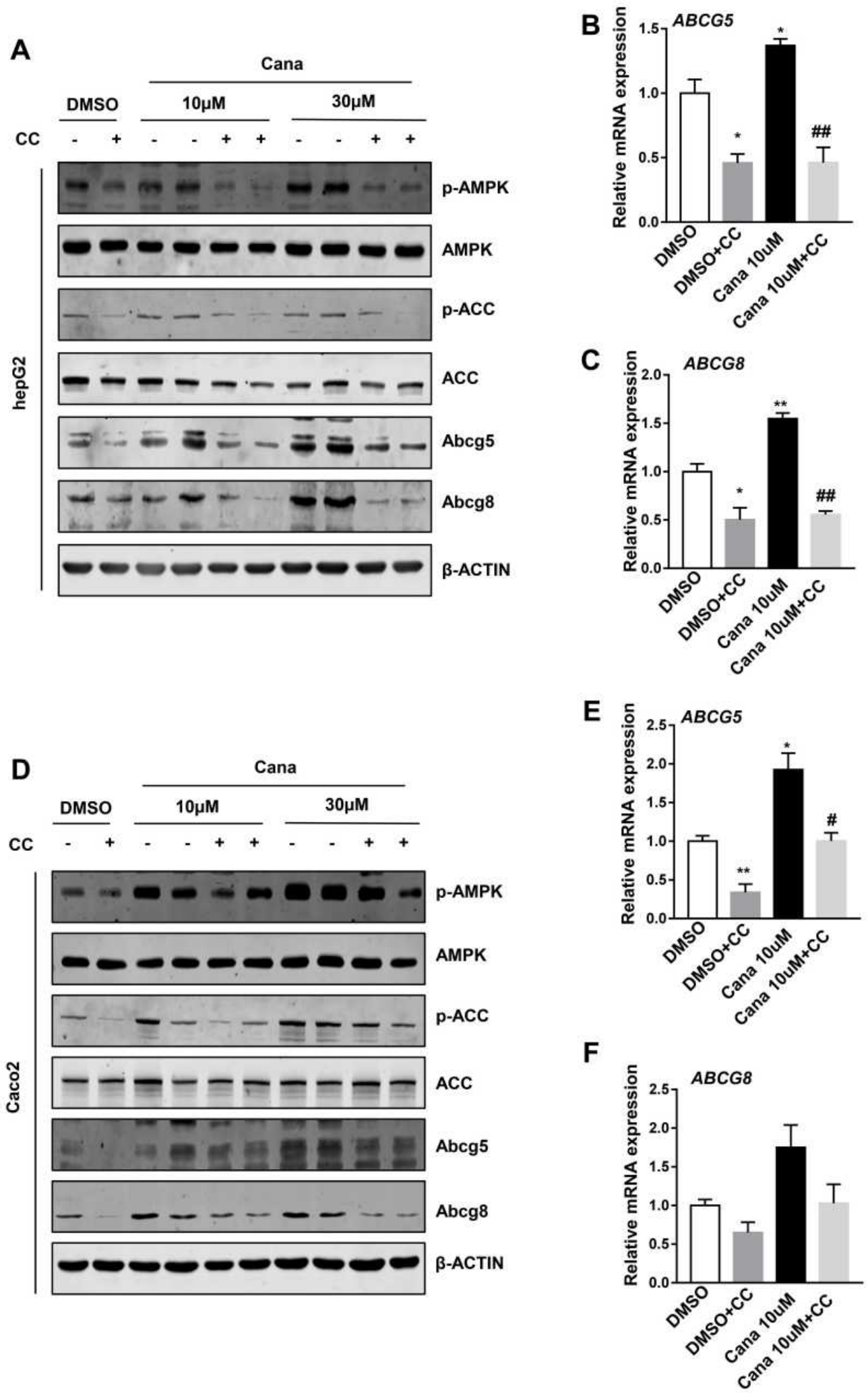

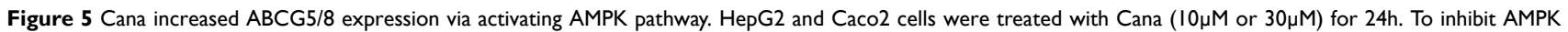

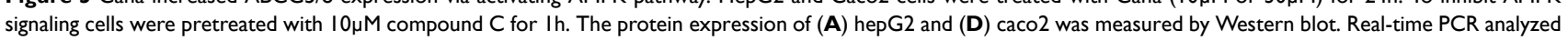
(B) ABCG5 and (C) ABCG8 expression in hepG2 cells, and (E) ABCG5 and (F) ABCG8 expression in caco2 cells. The data was shown with mean \pm SEM. *P<0.05, **P<0.01

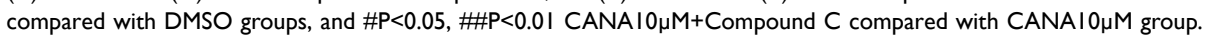
Abbreviation: $\mathrm{CC}$, compound $\mathrm{C}$. 
fecal excretion. Cana has been reported that inactive Hmgcr, a key rate-limiting enzyme in de novo synthesis of cholesterol. ${ }^{34}$ In our study, we also found Hmgcr and Hmgcs were decreased in Cana treatment mice. Abcg5 and Abcg8 form heterodimers acting as biliary and intestinal efflux pumps for dietary sterols and cholesterol on the apical side. ${ }^{35}$ Our study showed that Cana increased Abcg5 and Abcg8 expression in the liver and ileum facilitated cholesterol biliary and fecal excretion. Npc111 is the most important cholesterol absorption protein in the intestinal brush border membrane. ${ }^{5}$ Only increased Abcg5 and Abcg8, but not Npc111 can reduce atherosclerosis and improve cholesterol metabolism. ${ }^{7,36}$ Our study firstly reported that Cana could facilitate biliary and fecal cholesterol excretion but not change intestinal cholesterol absorption.

AMPK, an important energy sensor in cell growth, inhibits anabolic processes and promotes catabolism. Activating AMPK can inactivate Hmgcr, key enzyme of cholesterol synthesis. ${ }^{37}$ Fleur Lien reported that activation of AMPK can inhibit farnesoid X receptor (FXR) transcriptional activity, ${ }^{38}$ which is important in negativefeedback regulatory bile acid synthesis. Except Matthew, M. Molusky study found that AMPK through phosphorylate regulates Cry-1 and Per2 binding, which promotes ABCG5/8 expression. ${ }^{15}$ LXR is also an important cholesterol regulator. In macrophages, LXR agonist upregulates Abcal and Abag1 expression, and facilitates cholesterol conversion to HDL. ${ }^{39}$ In the liver and intestine, LXR is involved in the transcriptional control of Cyp7A1, as well as induction of $\mathrm{ABCA} 1, \mathrm{ABCG} 5$, and $\mathrm{ABCG} 8$ expression, thus accelerating biliary and fecal cholesterol disposal. ${ }^{24,28}$ And AMPK can also activate LXR and downstream proteins, upregulate cholesterol reverse transport. ${ }^{14}$ These results are coincident with our findings that Cana inhibits cholesterol synthesis, stimulates bile acid biosynthesis and facilities cholesterol efflux.

Cana has been certified to upregulate the ratio of AMP/ ADP, indirectly activating AMPK at lower dose and they confirmed that in mice $100 \mathrm{mg} / \mathrm{kg}$ Cana activated liver AMPK in $4 \mathrm{~h} .{ }^{29}$ In adipocyte, Cana promotes mitochondrial remodeling via AMPK-Sirt1-Pgc1 $\alpha$ pathway and stimulates energy metabolism. ${ }^{30}$ In vascular endothelial cells, Cana inhibits interleukin-1 $\beta$-stimulated secretion of IL-6 and monocyte chemoattractant protein- $1 .{ }^{40}$ However, there is no report about whether Cana activates AMPK in the intestinal segment. Therefore, we detected protein expression of AMPK and p-AMPK in duodenum, jejunum, and ileum. In the liver and intestine, Cana distinctly increased p-AMPK expression. ACC, a main major substrate of AMPK, can be phosphorylated and inhibited by AMPK. ${ }^{41}$ The phosphorylation of AMPK and its downstream protein ACC was enhanced by Cana. Thus, we used Compound $\mathrm{C}$, a pharmacological AMPK inhibitor, to inhibit AMPK in hepG2 cells and Caco2 cells. The expressions of Abcg5 and Abcg8 and LXR were also inhibited and Cana stimulating could not increase their expression. Therefore, AMPK activated by Cana is at least a reason for its effect on reverse cholesterol transport.

We have recognized the limitation of our study. AMPK activation can increase the expression of cholesterol efflux genes, imply antioxidant and anti-inflammatory capacities, thus been thought of as a therapeutic candidate for the treatment of cardiovascular disease. ${ }^{11,42}$ We found that inhibition of AMPK could abolish the effect of Cana which increased the expression of Abcg5/8. The genetic inhibition of AMPK in the liver or intestine could be used to further validate this effect. Cholesterol metabolism in macrophages is also an important part of reverse cholesterol transport. ${ }^{43}$ AMPK can also enhance the antiatherogenic properties of HDL. ${ }^{42}$ Cana's effect on macrophages can be studied further, and it may also be a reason of Cana improving cardiovascular outcomes.

In conclusion, we have shown the effect of Cana on blood lipid and hepatic cholesterol metabolism. Mechanically, we demonstrated that Cana activated LXR and increased Abcg5 and Abcg8 expression in liver and intestine via activating AMPK. Our results provide further evidence for clinical use of the drug in diabetic patients with high serum cholesterol or poor diet control.

\section{Abbreviations}

Abcg5/8, ATP-binding cassette transporters 5/8; AMPK, adenosine monophosphate-activated protein kinase; Abca1, ATP-binding cassette transporters A1; Abcg1, ATP-binding cassette transporters G1; HDL-C, highdensity lipoprotein-cholesterol; LDL-C, low-density lipoprotein-cholesterol; SGLT2, sodium glucose cotransporter 2; NPC1L1, Niemann-Pick C1-Like 1; HMGCR, 3-hydroxy-3-methylglutaryl-CoA reductase; HMGCS, 3-hydroxy-3-methylglutaryl-CoA synthase; LXR, liver $X$ receptor; FXR, farnesoid X receptor; ALT, serum alanine transaminase; AST, aspartate aminotransferase; $C D$, chow diet; WD, western diet; Cana, canagliflozin; CC, compound $\mathrm{C}$. 


\section{Acknowledgments}

We thank the Laboratory of Pathology, West China Hospital, for technical assistance.

\section{Author Contributions}

Y.Z. designed and performed experiments and wrote the manuscript. Y. L., Q. L., Q.T., J. Z., Z.Z. and C. H. contributed to the discussion and review of the manuscript. H.H., G.Z., J. Z., J.Y. and Y.X. helped with experiments. J. H. and Z.Z. obtained funding, designed experiments, and wrote the manuscript. All authors made substantial contributions to conception and design, acquisition of data, or analysis and interpretation of data; took part in drafting the article or revising it critically for important intellectual content; agreed to submit to the current journal; gave final approval for the version to be published; and agreed to be accountable for all aspects of the work.

\section{Funding}

This work was supported by the National Natural Science Foundation of China (81930020, 81603035 and 81870599), China Postdoctoral Fellowship (2017M612981), Young Scientist Fellowship of Sichuan University (2017SCU11026), and Postdoctoral Fellowship of Sichuan University (2017SCU12036).

\section{Disclosure}

The authors declare no conflict of interest. The funders had no role in the design of the study; in the collection, analyses, or interpretation of data; in the writing of the manuscript, or in the decision to publish the results.

\section{References}

1. Luo J, Yang H, Song BL. Mechanisms and regulation of cholesterol homeostasis. Nat Rev Mol Cell Biol. 2020;21:225-245.

2. Wang HH, Garruti G, Liu M, et al. Cholesterol and lipoprotein metabolism and atherosclerosis: recent advances in reverse cholesterol transport. Ann Hepatol. 2017;16:s27-s42. doi:10.5604/ 01.3001.0010.5495

3. Berbée J, Boon M, Khedoe P, et al. Brown fat activation reduces hypercholesterolaemia and protects from atherosclerosis development. Nat Commun. 2015;6(1):6356. doi:10.1038/ncomms7356

4. Bennion L, Grundy S. Effects of diabetes mellitus on cholesterol metabolism in man. $N$ Engl $J$ Med. 1977;296(24):1365-1371. doi:10.1056/NEJM197706162962401

5. Altmann S, Davis H, Zhu L, et al. Niemann-pick C1 like 1 protein is critical for intestinal cholesterol absorption. Science. 2004;303:1201-1204. doi:10.1126/science.1093131

6. Chiang JY. Bile acid metabolism and signaling. Compr Physiol. 2013;3:1191-1212. doi:10.1002/cphy.c120023
7. Basso F, Freeman LA, Ko C, et al. Hepatic ABCG5/G8 overexpression reduces apoB-lipoproteins and atherosclerosis when cholesterol absorption is inhibited. J Lipid Res. 2007;48(1):114-126. doi:10.1194/jlr.M600353-JLR200

8. Tontonoz P, Mangelsdorf DJ. Liver X receptor signaling pathways in cardiovascular disease. Mol Endocrinol. 2003;17(6):985-993. doi:10.1210/me.2003-0061

9. Teupser D, Kretzschmar D, Tennert C, et al. Effect of macrophage overexpression of murine liver $\mathrm{X}$ receptor-alpha (LXR-alpha) on atherosclerosis in LDL-receptor deficient mice. Arterioscler Thromb Vasc Biol. 2008;28:2009-2015. doi:10.1161/ATVBAHA.108.175257

10. Bujak AL, Crane JD, Lally JS, et al. AMPK activation of muscle autophagy prevents fasting-induced hypoglycemia and myopathy during aging. Cell Metab. 2015;21(6):883-890. doi:10.1016/j. cmet.2015.05.016

11. Carling D. AMPK signalling in health and disease. Curr Opin Cell Biol. 2017;45:31-37. doi:10.1016/j.ceb.2017.01.005

12. Fullerton MD, Ford RJ, McGregor CP, et al. Salicylate improves macrophage cholesterol homeostasis via activation of Ampk. J Lipid Res. 2015;56(5):1025-1033. doi:10.1194/jlr.M058875

13. Li CH, Gong D, Chen LY, et al. Puerarin promotes ABCA1-mediated cholesterol efflux and decreases cellular lipid accumulation in THP-1 macrophages. Eur J Pharmacol. 2017;811:74-86. doi:10.1016/j. ejphar.2017.05.055

14. Kemmerer M, Wittig I, Richter F, et al. AMPK activates LXR $\alpha$ and ABCA1 expression in human macrophages. Int J Biochem Cell Biol. 2016;78:1-9. doi:10.1016/j.biocel.2016.06.014

15. Molusky MM, Hsieh J, Lee SX, et al. Metformin and AMP kinase activation increase expression of the sterol transporters ABCG5/8 (ATP-binding cassette transporter G5/G8) with potential antiatherogenic consequences. Arterioscler Thromb Vasc Biol. 2018;38 (7):1493-1503. doi:10.1161/ATVBAHA.118.311212

16. Ji W, Zhao M, Wang M, et al. Effects of canagliflozin on weight loss in high-fat diet-induced obese mice. PLoS One. 2017;12(6): e0179960. doi:10.1371/journal.pone. 0179960

17. Stenlof K, Cefalu WT, Kim K-A, et al. Long-term efficacy and safety of canagliflozin monotherapy in patients with type 2 diabetes inadequately controlled with diet and exercise: findings from the 52-week CANTATA-M study. Curr Med Res Opin. 2014;30(2):163-175. doi:10.1185/03007995.2013.850066

18. Stenlof K, Cefalu WT, Kim K-A, et al. Efficacy and safety of canagliflozin monotherapy in subjects with type 2 diabetes mellitus inadequately controlled with diet and exercise. Diabetes Obes Metab. 2013;15(4):372-382. doi:10.1111/dom. 12054

19. Mahaffey K, Neal B, Perkovic V, et al. Canagliflozin for primary and secondary prevention of cardiovascular events: results from the CANVAS program (Canagliflozin Cardiovascular Assessment Study). Circulation. 2018;137(4):323-334. doi:10.1161/ CIRCULATIONAHA.117.032038

20. Xu C, Wang W, Zhong J, et al. Canagliflozin exerts anti-inflammatory effects by inhibiting intracellular glucose metabolism and promoting autophagy in immune cells. Biochem Pharmacol. 2018;152:45-59. doi:10.1016/j.bcp.2018.03.013

21. Kim SR, Lee SG, Kim SH, et al. SGLT2 inhibition modulates NLRP3 inflammasome activity via ketones and insulin in diabetes with cardiovascular disease. Nat Commun. 2020;11:2127. doi:10.1038/ s41467-020-15983-6

22. Devineni D, Polidori D. Clinical pharmacokinetic, pharmacodynamic, and drug-drug interaction profile of canagliflozin, a sodium-glucose co-transporter 2 Inhibitor. Clin Pharmacokinet. 2015;54:1027-1041. doi:10.1007/s40262-015-0285-z

23. Frambach S, de Haas R, Smeitink JAM, et al. Brothers in arms: ABCA1- and ABCG1-mediated cholesterol efflux as promising targets in cardiovascular disease treatment. Pharmacol Rev. 2020;72:152-190. doi:10.1124/pr.119.017897 
24. Wang B, Tontonoz P. Liver X receptors in lipid signalling and membrane homeostasis. Nat Rev Endocrinol. 2018;14(8):452-463. doi:10.1038/s41574-018-0037-x

25. Helgadottir A, Thorleifsson G, Alexandersson KF, et al. Genetic variability in the absorption of dietary sterols affects the risk of coronary artery disease. Eur Heart J. 2020;41(28):2618-2628. doi:10.1093/eurheartj/ehaa531

26. Castano D, Rattanasopa C, Monteiro-Cardoso VF, et al. Lipid efflux mechanisms, relation to disease and potential therapeutic aspects. $A d v$ Drug Deliv Rev. 2020;159:54-93.

27. He Y, Ronsein GE, Tang C, et al. Diabetes impairs cellular cholesterol efflux from ABCA1 to small HDL particles. Circ Res. 2020;127 (9):1198-1210. doi:10.1161/CIRCRESAHA.120.317178

28. Goedeke L, Rotllan N, Canfrán-Duque A, et al. MicroRNA-148a regulates LDL receptor and ABCA1 expression to control circulating lipoprotein levels. Nat Med. 2015;21(11):1280-1289. doi:10.1038/ nm.3949

29. Hawley SA, Ford RJ, Smith BK, et al. The Na+/glucose cotransporter inhibitor canagliflozin activates AMPK by inhibiting mitochondrial function and increasing cellular AMP levels. Diabetes. 2016;65 (9):2784-2794. doi:10.2337/db16-0058

30. Yang X, Liu Q, Li Y, et al. The diabetes medication canagliflozin promotes mitochondrial remodelling of adipocyte via the AMPKSirt1-Pgc-1 $\alpha$ signalling pathway. Adipocyte. 2020;9(1):484-494. doi:10.1080/21623945.2020.1807850

31. Nasiri-Ansari N, Dimitriadis GK, Agrogiannis G, et al. Canagliflozin attenuates the progression of atherosclerosis and inflammation process in APOE knockout mice. Cardiovasc Diabetol. 2018;17(1):106. doi:10.1186/s12933-018-0749-1

32. Yu T, Sungelo MJ, Goldberg IJ, et al. Streptozotocin-treated high fat fed mice: a new type 2 diabetes model used to Study Canagliflozin-Induced Alterations in Lipids and Lipoproteins. Horm Metab Res. 2017;49:400-406. doi:10.1055/s-0042-110934

33. Basu D, Huggins LA, Scerbo D, et al. Mechanism of increased LDL (Low-Density Lipoprotein) and decreased triglycerides with SGLT2 (Sodium-Glucose Cotransporter 2) inhibition. Arterioscler Thromb Vasc Biol. 2018;38:2207-2216. doi:10.1161/ATVBAHA.118.311339
34. Day EA, Ford RJ, Lu JH, et al. The SGLT2 inhibitor canagliflozin suppresses lipid synthesis and interleukin-1 beta in ApoE deficient mice. Biochem J. 2020;477:2347-2361. doi:10.1042/BCJ20200278

35. Wang HH, Li T, Portincasa P, et al. New insights into the role of Lith genes in the formation of cholesterol-supersaturated bile. Liver Res. 2017;1(1):42-53. doi:10.1016/j.livres.2017.05.005

36. Wilund KR, Yu L, Xu F, et al. High-level expression of ABCG5 and ABCG8 attenuates diet-induced hypercholesterolemia and atherosclerosis in Ldlr-- mice. J Lipid Res. 2004;45(8):1429-1436. doi:10.1194/jlr.M400167-JLR200

37. González A, Hall MN, Lin S-C, et al. AMPK and TOR: the Yin and Yang of cellular nutrient sensing and growth control. Cell Metab. 2020;31:472-492.

38. Lien F, Berthier A, Bouchaert E, et al. Metformin interferes with bile acid homeostasis through AMPK-FXR crosstalk. J Clin Invest. 2014;124(3):1037-1051. doi:10.1172/JCI68815

39. Lefterov I, Bookout A, Wang Z, et al. Expression profiling in APP23 mouse brain: inhibition of $\mathrm{A} \beta$ amyloidosis and inflammation in response to LXR agonist treatment. Mol Neurodegener. 2007;2 (1):20. doi:10.1186/1750-1326-2-20

40. Mancini SJ, Boyd D, Katwan OJ, et al. Canagliflozin inhibits interleukin-1 $\beta$-stimulated cytokine and chemokine secretion in vascular endothelial cells by AMP-activated protein kinase-dependent and independent mechanisms. Sci Rep. 2018;8(1):5276. doi:10.1038/ s41598-018-23420-4

41. Qiang L, Sample A, Shea CR, et al. Autophagy gene ATG7 regulates ultraviolet radiation-induced inflammation and skin tumorigenesis. Autophagy. 2017;13(12):2086-2103. doi:10.1080/15548627. 2017.1380757

42. Ma A, Wang J, Yang L, et al. AMPK activation enhances the antiatherogenic effects of high density lipoproteins in apoE-/- mice. J Lipid Res. 2017;58(8):1536-1547. doi:10.1194/jlr.M073270

43. Cedo L, Metso J, Santos D, et al. LDL receptor regulates the reverse transport of macrophage-derived unesterified cholesterol via concerted action of the HDL-LDL axis: insight from mouse models. Circ Res. 2020;127:778-792. doi:10.1161/CIRCRESAH A.119.316424
Drug Design, Development and Therapy

\section{Publish your work in this journal}

Drug Design, Development and Therapy is an international, peerreviewed open-access journal that spans the spectrum of drug design and development through to clinical applications. Clinical outcomes, patient safety, and programs for the development and effective, safe, and sustained use of medicines are a feature of the journal, which has also been accepted for indexing on PubMed Central. The manuscript management system is completely online and includes a very quick and fair peer-review system, which is all easy to use. Visit http://www. dovepress.com/testimonials.php to read real quotes from published authors. 